\title{
Microencapsulation of herbicide MCPA with native $\beta$-cyclodextrin and its methyl and hydroxypropyl derivatives: An experimental and theoretical investigation
}

\author{
Jorge Garrido ${ }^{\mathrm{a}, \mathrm{b}, *}$, Fernando Cagide ${ }^{\mathrm{b}}$, Manuel Melle-Franco ${ }^{\mathrm{c}}$, Fernanda Borges $^{\mathrm{b}}$, E. Manuela Garrido ${ }^{\mathrm{a}, \mathrm{b}}$ \\ ${ }^{a}$ Departamento de Engenharia Química, Instituto Superior de Engenharia do Porto (ISEP), Instituto Politécnico do Porto, $4200-072$ Porto, Portugal \\ ${ }^{\mathrm{b}} \mathrm{CIQ}$ Departamento de Química e Bioquímica, Faculdade de Ciências, Universidade do Porto, 4169-007 Porto, Portugal \\ ${ }^{\mathrm{C}}$ CCTC, Departamento de Informática, Universidade do Minho, 4710-057 Braga, Portugal
}

\section{H I G H L I G H T S}

- Microencapsulation of herbicide MCPA with different $\beta$-CDs has been investigated.

- Formation of the inclusion complexes increased the water solubility of MCPA.

- ${ }^{1} \mathrm{H}$ NMR data obtained provided clear evidence of inclusion complexation process.

- Computer models were used to compare different $\beta$-CDs binding affinity for MCPA.

\section{A R T I C L E I N F O}

\section{Article history:}

Received 20 September 2013

Received in revised form 18 December 2013

Accepted 19 December 2013

Available online 3 January 2014

\section{Keywords:}

Phenoxyacetic acid herbicides

MCPA

$\beta$-Cyclodextrin

Inclusion complex

\begin{abstract}
A B S T R A C T
When a pesticide is released into the environment, most of it is lost before it reaches its target. An effective way to reduce environmental losses of pesticides is by using controlled release technology. Microencapsulation becomes a promising technique for the production of controlled release agricultural formulations. In this work, the microencapsulation of chlorophenoxy herbicide MCPA with native $\beta$-cyclodextrin and its methyl and hydroxypropyl derivatives was investigated. The phase solubility study showed that both native and $\beta-C D$ derivatives increased the water solubility of the herbicide and inclusion complexes are formed in a stoichiometric ratio of $1: 1$. The stability constants describing the extent of formation of the complexes have been determined by phase solubility studies. ${ }^{1} \mathrm{H}$ NMR experiments were also accomplished for the prepared solid systems and the data gathered confirm the formation of the inclusion complexes. ${ }^{1} \mathrm{H}$ NMR data obtained for the MCPA/CDs complexes disclosed noticeable proton shift displacements for $\mathrm{OCH}_{2}$ group and $\mathrm{H} 6$ aromatic proton of MCPA provided clear evidence of inclusion complexation process, suggesting that the phenyl moiety of the herbicide was included in the hydrophobic cavity of CDs. Free energy molecular mechanics calculations confirm all these findings.

The gathered results can be regarded as an essential step to the development of controlled release agricultural formulations containing herbicide MCPA.
\end{abstract}

(c) 2014 Elsevier B.V. All rights reserved.

\section{Introduction}

The world's population is set to grow considerably over the coming years, albeit at a slower rate than in the past, and with considerable differences across regions [1]. Over the next four decades, the world's population is forecast to increase by 2 billion people to exceed 9 billion people by 2050. Recent FAO estimates indicate that to meet the projected demand, global agricultural production

\footnotetext{
* Corresponding author at: Departamento de Engenharia Química, Instituto Superior de Engenharia do Porto (ISEP), Instituto Politécnico do Porto, 4200-072 Porto, Portugal. Tel.: +351 228340500.

E-mail address: jjg@isep.ipp.pt (J. Garrido).
}

will have to increase by $60 \%$ from its 2005-2007 levels [1]. However, the intensification of production can be associated with significant negative environmental effects, including groundwater pollution, soil erosion and a loss in biodiversity. Some $20-40 \%$ of the world's potential crop production is already lost annually because of the effects of weeds, pests and diseases [2]. The use of pesticides has been one of the main tools to combat a variety of pests that could destroy crops and to improve the quality of the food produced. However, the adverse impacts of these compounds on the environment and ecosystems cannot be ignored. In fact, pesticides can have a significant negative environmental effect, by contaminating soil, surface and ground water, and contribute to a loss 
in biodiversity. Therefore, the growing concern about environmental protection, human health, and food safety has brought renewed interest in pesticide use in agriculture.

Controlled release formulations give a high promise for enhancing the efficacy of biological active agents in the agrochemical domain, since they can contribute to diminish the harmful effects of pesticides and to reduce their environmental pollution [3-6]. Controlled release pesticide formulations can maintain the concentration threshold of the active ingredient which controls the pest in the soil or plant for longer by releasing it at an appropriate speed, thereby reducing its level in the environment because lower amounts or fewer applications are required in order to achieve the pursued biological effect. Microencapsulation becomes one of the most important industrial processes used for the production of controlled release agricultural formulations [7-9]. The main objective of encapsulation is to protect the core material from adverse environmental conditions, such as undesirable effects of light, moisture, and oxygen, thereby contributing to an increase in the shelf life of the product, and promoting a controlled liberation of the encapsulate [10].

Cyclodextrins, CDs, are macrocyclic oligosugars most commonly composed of 6,7 , or 8 glucosidic units bearing the names $\alpha-1, \beta-2$ and $\gamma-3 \mathrm{CD}$, respectively [11]. Among them, $\beta-C D$ is receiving increasing attention due its low cost and high capacity to interact with a wide variety of molecules, including pesticides and drugs. Chemically modified $C D$ derivatives have been prepared with a view to extend the physicochemical properties and inclusion capacity of parent CDs. Cyclodextrins have been used for the molecular encapsulation in a broad variety of applications, especially in pharmaceutical and environmental chemistry, because these types of compounds are very convenient due to their great variability of molecular shape and molecular properties. CDs, with lipophilic inner cavities and hydrophilic outer surfaces, are capable of interacting with a large variety of guest molecules to form noncovalent inclusion complexes.

As a part of an extended project aimed to validate the application of nanotechnology for chlorophenoxy herbicides management an experimental and theoretical study of the inclusion effect of native $\beta-C D$ and its methyl and hydroxypropyl derivatives on the properties of MCPA (4-chloro-2-methylphenoxyacetic acid) has been performed. MCPA is in the top five of the most commonly used pesticide active ingredients in the home and garden sector in the US and herbicide active ingredients in the EU and still remain one of the most often used herbicides for rice crops in Portugal [12,13]. It has been described that the formation of an inclusion complex between MCPA and CDs increased the aqueous solubility of this herbicide $[9,14,15]$. In the present study the influence of the pattern of substitution and reactivity of different $\beta-C D$ on the quality of the interactions and stability of MCPA inclusion complexes is evaluated with the aim to contribute to the future applications of CDs namely to improve commercial formulation and for environmental protection.

\section{Materials and methods}

\subsection{Chemicals}

MCPA, $\beta$-cyclodextrin, (2-hydroxypropyl)- $\beta$-cyclodextrin $(0.8$ molar substitution) and methyl- $\beta$-cyclodextrin (1.6-2.0 $\mathrm{mol} \mathrm{CH}_{3}$ per unit anhydroglucose) were purchased from Sigma-Aldrich Química S.A. (Sintra, Portugal). Deuterated solvents and tetramethylsilane (TMS) were obtained from Merck (Lisbon, Portugal). All other reagents and solvents were pro analysis grade and used without additional purification. Deionised water (conductivity $<0.1 \mu \mathrm{S} \mathrm{cm}^{-1}$ ) was used throughout all the experiments.

\subsection{Phase solubility studies}

Phase solubility studies were carried out according to the method described by Higuchi and Connors [16]. Excess amount of MCPA $(25 \mathrm{mg}$ ) was added to $7.5 \mathrm{~mL}$ of aqueous solutions containing various concentrations of $\beta-\mathrm{CD}(0-9 \mathrm{mM})$ and (2-hydroxypropyl)$\beta$-cyclodextrin and methyl- $\beta$-cyclodextrin (0-35 mM). Then, the suspensions were shaken on a rotary shaker at $25 \pm 2{ }^{\circ} \mathrm{C}$ for 4 days. After equilibrium was reached, suspensions were centrifuged and the supernatant was withdrawn and properly diluted. The concentration of MCPA was determined by spectrophotometry (Shimadzu UV-Vis Spectrophotometer, UV-1700 PharmaSpec, Japan) at $228 \mathrm{~nm}$. The apparent stability constants $K_{\mathrm{s}}$ were calculated from phase solubility diagrams with the assumption of $1: 1$ stoichiometry according to Eq. (1):

$K_{s}=$ slope $/ S_{0}(1-$ slope $)$

$S_{0}$ is the solubility of MCPA in absence of CDs.

\subsection{Preparation of the complexes}

The preparation of MCPA/ $\beta$-cyclodextrin ( $\beta$-CD), (2-hydroxypropyl)- $\beta$-cyclodextrin (HP- $\beta$-CD) and methyl- $\beta$-cyclodextrin (Me- $\beta-C D$ ) inclusion complexes has been performed by a co-evaporation procedure as described elsewhere [9]. Briefly, MCPA and each of the CDs under study (equimolar ratio) were completely dissolved in a solution of ethanol and water $(\mathrm{v} / \mathrm{v}=1: 20)$. The dispersion of MCPA in the aqueous CDs solutions was protected from light and mechanically shaken at room temperature and $100 \mathrm{rpm}$ in an IKA KS 4000i incubator shaker (IKA, Germany) for $48 \mathrm{~h}$ to achieve equilibrium of the complexation reaction. After evaporation of the ethanol from the reaction mixture, the uncomplexed MCPA was removed by filtration. The filtrate was evaporated under reduced pressure in a Büchi Rotavapor (Büchi, Germany) to remove the solvent and dried in vacuum to give the MCPA/ $\beta-C D, M C P A / H P-$ $\beta-\mathrm{CD}$ and MCPA/Me- $\beta-\mathrm{CD}$ complexes.

\subsection{Physicochemical characterisation of MCPA-CD complexes}

\subsubsection{UV-Vis spectroscopy}

Spectrophotometric measurements were performed to quantify MCPA in its free and CD-complexed form. Standard curves of MCPA were prepared in deionised water.

Complete spectrophotometric scans between 190 and $300 \mathrm{~nm}$ were performed to monitor any changes in the UV spectra of the MCPA. The absorbance maxima of $228 \mathrm{~nm}$, was used to quantify MCPA concentration.

\subsection{Nuclear magnetic resonance studies}

${ }^{1} \mathrm{H}$ NMR data were acquired at room temperature and recorded on a Bruker Avance III operating at $400 \mathrm{MHz}$. Chemical shifts are expressed in $\delta(\mathrm{ppm})$ values relative to tetramethylsilane (TMS) as internal reference. Chemical shifts changes $(\Delta \delta)$ were calculated according to the formula $\Delta \delta=\delta_{\text {(complex) }}-\delta_{\text {(free) }}$.

All NMR experiments were carried out in deuterated water $\left(\mathrm{D}_{2} \mathrm{O}\right)$, except for MCPA. Owing to MCPA extremely poor aqueous solubility the spectra was acquired in deuterated methanol $\left(\mathrm{CD}_{3} \mathrm{OD}\right)$. The proton chemical shifts of MCPA obtained in $\mathrm{CD}_{3} \mathrm{OD}$ are quite similar to that found in literature using $\mathrm{D}_{2} \mathrm{O}$ as solvent [17].

\subsection{Computer modelling}

Molecular mechanics simulations were performed in order to model the effect of the pattern of substitution (functional groups) 
in the molecular recognition between MCPA and the different CDs under study. All calculations were done with the Tinker software suite [18] with the Optimized Potentials for Liquid Simulations (OPLS) [19] combined with the TIP3P model for explicit water [20]. OPLS was chosen as it includes parameters specifically derived for carbohydrates. Implicit solvent calculations were computed with the Generalized Born model augmented with an accessible Surface Area term (GBSA) [21,22].

\section{Results and discussion}

In aqueous solutions cyclodextrins are able to form inclusion complexes with many drugs by taking up a drug molecule, or more frequently some lipophilic moiety of the molecule, into the central cavity. No covalent bonds are formed or broken during the complex formation, and drug molecules in the complex are in rapid equilibrium with free molecules in the solution [23].

The physicochemical properties of free drug molecules are different from those bound to the cyclodextrin molecules. Methodologies that can be used to observe these changes in additive physicochemical properties may be utilised to determine the stoichiometry of the complexes formed and the numerical values of their stability constants [23]. These include changes, for instance, in solubility, UV/Vis absorbance and nuclear magnetic resonance (NMR) chemical shifts.

\subsection{Phase solubility studies}

The formation of inclusion complexes MCPA/CDs was confirmed by UV-Vis spectrophotometry. The stoichiometric ratio and stability constants were derived from the changes in the solubility of MCPA in the presence of increasing amounts of $\beta-C D$, HP$\beta-C D$ and Me- $\beta-C D$. The phase solubility plots obtained at $25^{\circ} \mathrm{C}$ are shown in Fig. 1 . As can be seen, in all cases $A_{L}$-type solubility diagrams were obtained as the MCPA solubility increased linearly with the increment of $\beta-C D, H P-\beta-C D$ and Me- $\beta-C D$ concentration, according to the classification established by Higuchi and Connors [16]. The diagrams obtained indicate that the solubility of the MCPA is significantly increased by the presence of the macrocycles.

Table 1 summarizes the MCPA solubility, slope, stability constant and correlation coefficient of the phase solubility diagrams for the different cyclodextrins under study. Since the slope of the diagrams is less than 1 , the complex stoichiometry was assumed to be $1: 1$. The stability constants, $K_{\mathrm{s}}$, were therefore calculated

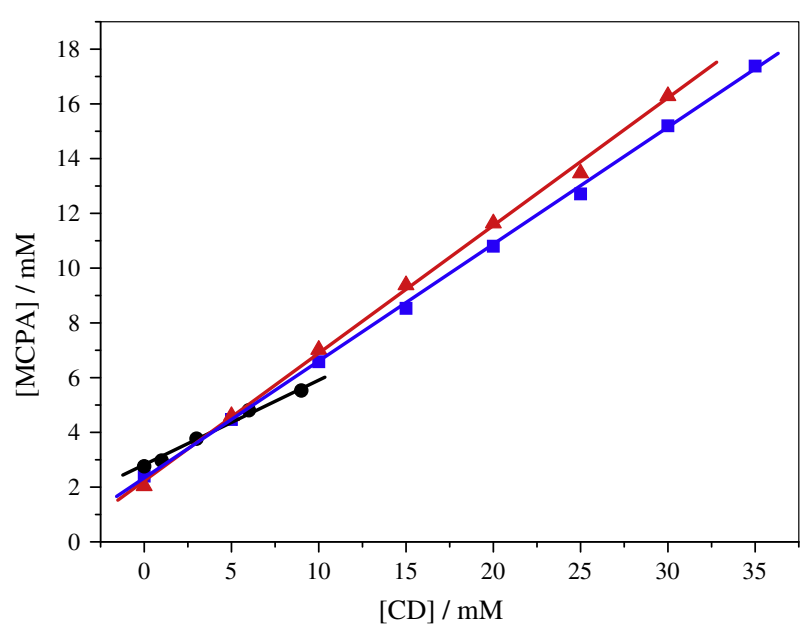

Fig. 1. Phase solubility diagram for MCPA and increasing concentrations of $(\bullet,-)$ $\beta-C D,(\square,-)$ HP- $\beta-C D$ and $(\Delta,-)$ Me- $\beta-C D$ in water at $25^{\circ} \mathrm{C}$. Each point represents the mean of three determinations.
Table 1

Solubility of MCPA $\left(S_{0}\right)$, slope $(\alpha)$, stability constant $\left(K_{\mathrm{s}}\right)$ and correlation coefficient $\left(R^{2}\right)$ in the different cyclodextrins.

\begin{tabular}{lllll}
\hline & $\mathrm{S}_{0} \pm \mathrm{SD}^{\mathrm{a}}\left(\times 10^{-3} \mathrm{M}\right)$ & $\alpha \pm \mathrm{SD}^{\mathrm{a}}$ & $K_{\mathrm{s}} \pm \mathrm{SD}^{\mathrm{a}}\left(\mathrm{M}^{-1}\right)$ & $R^{2}$ \\
\hline$\beta-\mathrm{CD}$ & $0.00272 \pm 5 \times 10^{-5}$ & $0.30 \pm 0.05$ & $162 \pm 12$ & 0.998 \\
$\mathrm{HP}-\beta-\mathrm{CD}$ & $0.00222 \pm 4 \times 10^{-5}$ & $0.45 \pm 0.03$ & $334 \pm 9$ & 0.999 \\
Me- $\beta-C D$ & $0.00232 \pm 2 \times 10^{-5}$ & $0.45 \pm 0.01$ & $398 \pm 13$ & 0.998 \\
\hline
\end{tabular}

a Standard deviations were calculated on triplicate trials.

from the straight-line portion of the phase solubility diagrams, according to the equation previously presented (see experimental). Stability constants calculated for $\beta-C D, H P-\beta-C D$ and Me- $\beta-C D$ were $162 \pm 12,334 \pm 9$ and $398 \pm 13 \mathrm{M}^{-1}$, respectively. As can be seen, the stability constants calculated for HP- $\beta-C D$ and Me- $\beta-C D$ systems are two fold greater than that obtained for the $\beta-C D$ indicating that these complexes are more stable. The higher association constant values obtained using HP- $\beta-C D$ and Me- $\beta-C D$ were expected considering that the polarity of the substituents of the host could contribute to the complex formation. Thus less polar groups like 2-hydroxypropyl or methyl can be more favourable to the formation of the complex [24].

\subsection{Nuclear Magnetic Resonance analysis (NMR)}

Various known methods have been used for the formation of the inclusion complexes. The selection of the method of preparation of the inclusion compounds and its effectiveness highly depends on the nature of the drug and cyclodextrin [9]. The preparation of MCPA/CDs solid systems was performed using the co-evaporation method.

One-dimensional proton nuclear magnetic resonance spectroscopy ( ${ }^{1} \mathrm{H}$ NMR) analysis was employed to verify and characterize the inclusion of the MCPA in the CDs under study. NMR is a powerful and simple technique that can be used in the study of the inclusion process of a guest in the hydrophobic cavity of CDs. ${ }^{1} \mathrm{H}$ NMR can provide unique structural information about the inclusion of a guest into the cavity of the CDs and the stoichiometry of the system. Through the proton chemical shift displacements directly involved in the encapsulation process important information about the formation of the complexes can be attained [25].

Accordingly, ${ }^{1} \mathrm{H}$ NMR data was acquired to confirm the formation of the inclusion complexes of the herbicide MCPA with different types of CDs. The spectra of MCPA, CDs (HP- $\beta-C D$ and Methyl- $\beta-\mathrm{CD}$ ) and the inclusion complexes (MCPA/HP- $\beta-\mathrm{CD}$ and MCPA/Methyl- $\beta-C D$ ) are shown in Figs. 2 and 3. The NMR data related to the MCPA $\beta-C D$ complex has been reported in a previous study [9]. The numbering of the hydrogen atoms faced inside and outside of the CDs cavity has been ascribed in accordance with the literature [26].

As the formation of host-guest inclusion complexes induces variable levels of changes on the chemical shifts $(\delta)$ of the hydrogens either of the ligand or the $\mathrm{CD}$, the chemical displacement of the proton signals of MPCA/CDs complexes with respect to the native samples per si (MCPA and the corresponding CDs) have been determined. The chemical shift data $(\delta)$ of MCPA and HP- $\beta-C D$ before and after the formation of inclusion complex are listed in Table 2 . The upfield shifted proton signals, with respect to the native compounds, are caused by anisotropic effects and indicate the presence of host-guest interactions in the CD cavity.

From data analysis one can conclude MCPA protons undergo upfield displacements in the presence of cyclodextrin, namely $\mathrm{H} 6$ and $\mathrm{CH}_{2}$ protons due probably to host-guest interactions. The previous assumptions are also sustained by the noticeably displacements observed for the $\mathrm{H} 3^{\prime}, \mathrm{H} 5^{\prime}$ and $\mathrm{H} 6^{\prime}$ protons of $\mathrm{HP}-\beta-\mathrm{CD}$ 

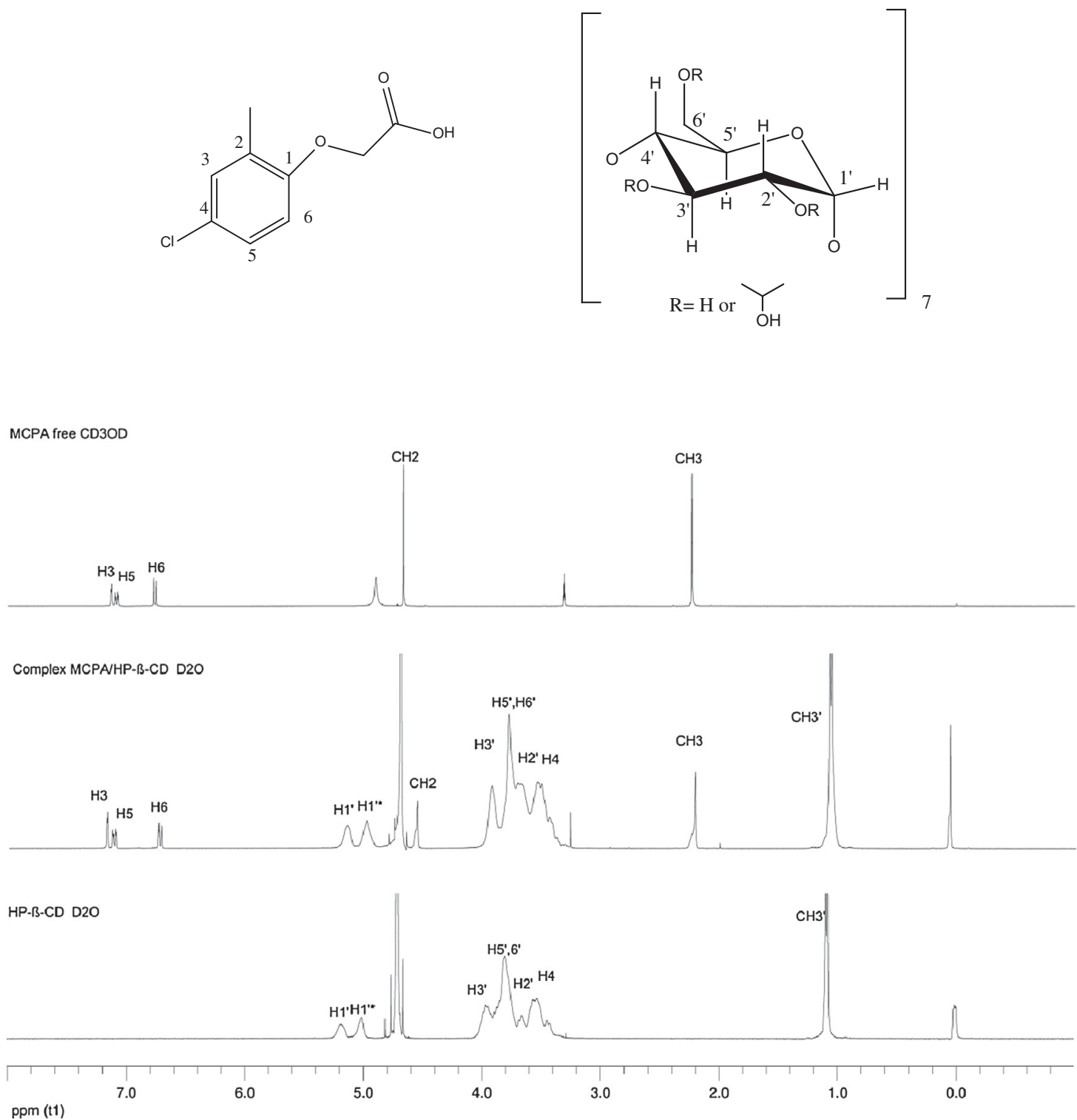

Fig. 2. ${ }^{1} \mathrm{H}$ NMR spectra of MCPA, HP- $\beta-C D$ and $M C P A / H P-\beta-C D$ inclusion complex.

cavity. This effect could be as well a consequence of the occurrence of a strong interaction between the host (MCPA) and the guest (HP$\beta-C D)$. In summary the data clearly indicate the insertion of the aromatic part of MCPA into the CD cavity.

Similar results were obtained for the complex MPCA/Methyl- $\beta$ cyclodextrin. Significant upfield chemical shift variations of proton signals in the complex with respect to the native compounds have been also detected (Table 3). Noticeable upfield shift displacements were also observed for $\mathrm{OCH}_{2}$ group and the $\mathrm{H} 6$ aromatic proton of MCPA.

The stoichiometry of the complexes has been also determined by comparing the ${ }^{1} \mathrm{H}$ NMR integration data between the $\mathrm{H} 6$ proton in MPCA and $\mathrm{H}^{\prime}{ }^{\prime}$ proton of the corresponding $\mathrm{CD}$. For the MPCA/HP$\beta-C D$ and MPCA/Methyl- $\beta-C D$ complexes a relationship of approximately $1: 7$ was obtained corresponding to a $1: 1$ stoichiometry.

\subsection{Computer modelling}

Computer models have been extensively used in order to understand inclusion complexes of functionalized and pure $\beta$-CDs [27]. Thus, molecular mechanics simulations have been performed to understand the increased solubility/binding of MCPA with functionalized $\beta$-CDs.

Two different methylated $\beta$-CDs with one and two methyl groups per anhydroglucose unit have been studied; 7Me- $\beta-C D$ $[28]$ and $14 \mathrm{Me}-\beta-\mathrm{CD}$ (heptakis (2, 6-di-O-methyl)- $\beta$-cyclodextrin) [29], respectively. In order to calculate the binding energies differences the free energy of solvation of seven different systems were computed, namely: MCPA, $\beta-C D, 7 \mathrm{Me}-\beta-\mathrm{CD}, 14 \mathrm{Me}-\beta-\mathrm{CD}, \mathrm{MCPA}-\beta-$ CD, MCPA-7Me- $\beta-C D$ and MCPA- 14 Me- $\beta-C D$. Firstly systems were solvated inside a cubic box of water with $\sim 4 \mathrm{~nm}$ side. Secondly, 7 nanoseconds of NPT molecular dynamics simulations were run with 1 femtosecond time steps at room temperature. The final step involved analyzing the energetics of snapshot conformations at 1 picosecond intervals with an implicit solvent model. MCPA-7Me$\beta$-CD and MCPA-14Me- $\beta$-CD complexes yielded a $1.8 \mathrm{kcal} / \mathrm{mol}$ increase in binding energy with respect to MCPA- $\beta-C D$. Entropy and zero point energy corrections were also calculated but yielded quite similar values for $\beta-C D$ and $\mathrm{Me}-\beta-\mathrm{CD}$ and will not be discussed here. Similar energetics has been obtained in a previous study of cholesterol complexed with $14 \mathrm{Me}-\beta-\mathrm{CD}$ and HP- $\beta-\mathrm{CD}[30]$. The lowest energy configurations for MCPA- $\beta-C D$ and MCPA$14 \mathrm{Me}-\beta-\mathrm{CD}$ complexes are represented in Fig. 4. Interestingly, the 

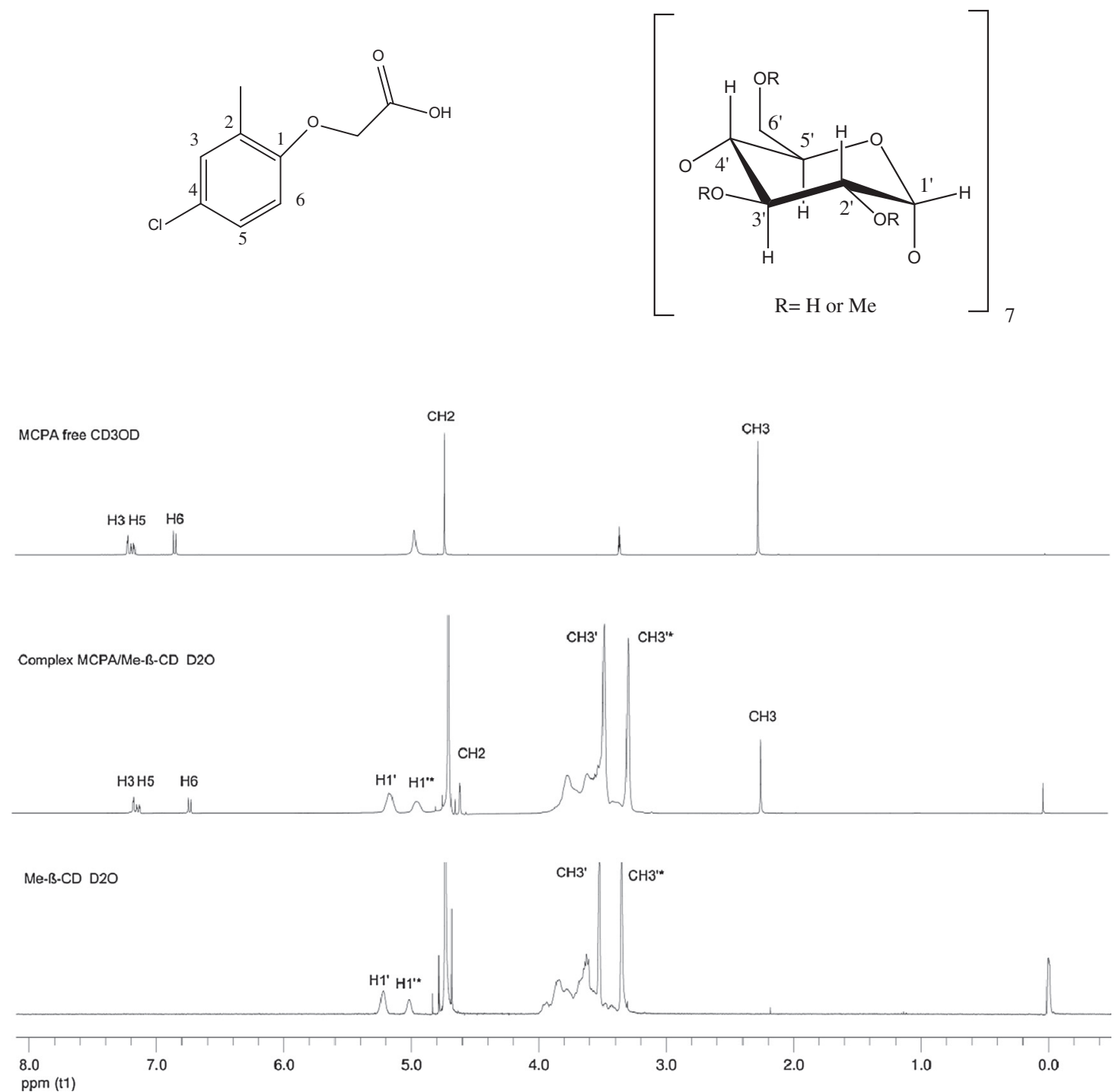

Fig. 3. ' $\mathrm{H}$ NMR spectra of MCPA, methyl- $\beta-C D$ and $M C P A /$ methyl- $\beta-C D$ inclusion complex.

Table 2

${ }^{1} \mathrm{H}$ NMR chemical shift $(\delta)$ data of MCPA, HP- $\beta-C D$ and MCPA/HP- $\beta-C D$ complex.

\begin{tabular}{lllll}
\hline $\mathrm{H}$ & $\mathrm{MCPA}$ & $\mathrm{HP}-\beta-\mathrm{CD}$ & $\mathrm{MCPA} / \mathrm{HP}-\beta-\mathrm{CD}$ & $\Delta \delta$ \\
Assignment & $\left(\mathrm{CD}_{3} \mathrm{OD}\right)$ & $\left(\mathrm{D}_{2} \mathrm{O}\right)$ & $\left(\mathrm{D}_{2} \mathrm{O}\right)$ & \\
\hline $\mathrm{H} 3$ & 7.129 & - & 7.084 & -0.045 \\
$\mathrm{H} 5$ & 7.087 & - & 7.032 & -0.055 \\
$\mathrm{H} 6$ & 6.761 & - & 6.648 & -0.113 \\
$\mathrm{CH}_{2}$ & 4.666 & - & 4.524 & -0.142 \\
$\mathrm{CH}_{3}$ & 2.232 & - & 2.210 & -0.022 \\
$\mathrm{H}^{\prime}$ & - & 5.180 & 5.064 & -0.109 \\
$\mathrm{H}^{\prime}$ & - & 5.003 & 4.893 & -0.110 \\
$\mathrm{H}^{\prime}$ & - & 3.939 & 3.836 & -0.103 \\
$\mathrm{H}^{\prime}$ & - & 3.791 & 3.693 & -0.098 \\
$\mathrm{H}^{\prime}$ & - & 3.791 & 3.693 & -0.098 \\
$\mathrm{H}^{\prime}$ & - & 3.660 & 3.595 & -0.065 \\
$\mathrm{H}^{\prime}$ & - & 3.534 & 3.438 & -0.096 \\
$\mathrm{CH}_{3}{ }^{\prime}$ & - & 1.074 & 0.980 & 0.094 \\
\hline
\end{tabular}

orientation of MCPA differs: for MCPA- $\beta-C D$ the carboxylic group sticks out from the narrower side of $\beta$-CD (Fig. 4a) while for MCPA-14Me- $\beta-C D$ the carboxylic group points into the opposite direction (Fig. 4b). Summing up, MCPA flips orientation when bound to functionalized $\beta$-CDs increasing the stability of the
Table 3

${ }^{1} \mathrm{H}-\mathrm{NMR}$ chemical shift $(\delta)$ data of MCPA, Methyl- $\beta-C D$ and MCPA/Methyl- $\beta-C D$ complex.

\begin{tabular}{lllll}
\hline $\begin{array}{l}\mathrm{H} \\
\text { Assignment }\end{array}$ & $\begin{array}{l}\text { MCPA } \\
\left(\mathrm{CD}{ }_{3} \mathrm{OD}\right)\end{array}$ & $\begin{array}{l}\text { Methyl- } \beta-\mathrm{CD} \\
\left(\mathrm{D}_{2} \mathrm{O}\right)\end{array}$ & $\begin{array}{l}\text { MCPA/Methyl- } \beta-\mathrm{CD} \\
\left(\mathrm{D}_{2} \mathrm{O}\right)\end{array}$ & $\Delta \delta$ \\
\hline $\mathrm{H} 3$ & 7.129 & - & 7.054 & -0.075 \\
$\mathrm{H} 5$ & 7.087 & - & 7.021 & -0.066 \\
$\mathrm{H} 6$ & 6.761 & - & 6.619 & -0.142 \\
$\mathrm{CH}_{2}$ & 4.666 & - & 4.523 & -0.143 \\
$\mathrm{CH}_{3}$ & 2.232 & - & 2.192 & -0.040 \\
$\mathrm{H} 1$ & - & 5.220 & 5.074 & -0.156 \\
$\mathrm{H} 1^{\prime}$ & - & 5.018 & 4.862 & -0.151 \\
$\mathrm{CH}_{3}{ }^{\prime}$ & - & 3.521 & 3.405 & -0.116 \\
$\mathrm{CH}_{3}{ }^{\prime}$ & - & 3.350 & 3.219 & -0.131 \\
\hline
\end{tabular}

resulting complexes. This finding confirms the molecular origin of the experimental observations.

\section{Conclusion}

The native CDs can be chemically modified by hydroxyalkylation, alkylation or sulfoalkylation. The principal aim of such modification is to increase the solubility and safety profile of the 

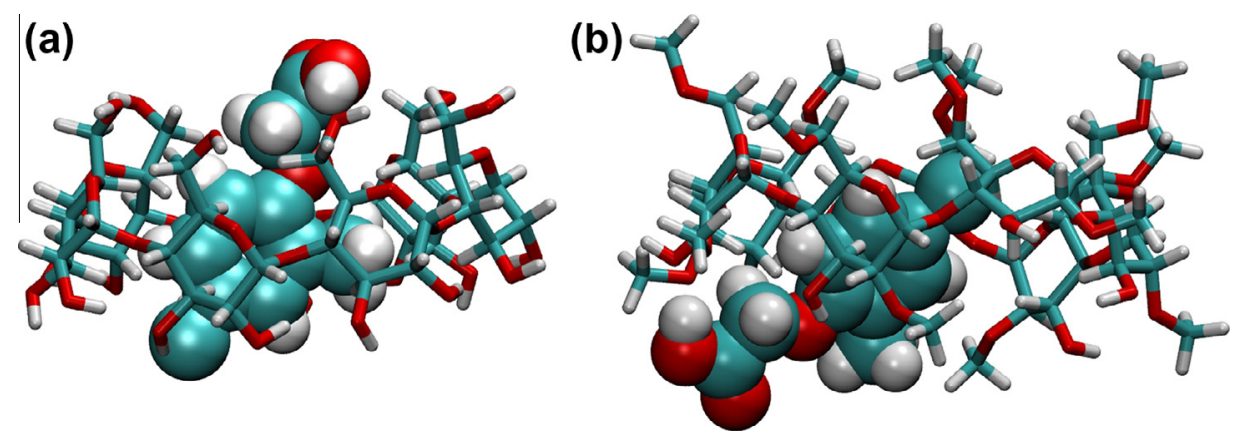

Fig. 4. Molecular models of MCPA (sphere model) inside (a) $\beta-C D$ and (b) $14 \mathrm{Me}-\beta-\mathrm{CD}$ (stick model).

parent native cyclodextrins. In recent years, $\beta-C D$ has gained appreciable acceptance amongst the various types of cyclodextrins. The high aqueous solubility of modified cyclodextrins such as HP$\beta-C D$ and Me- $\beta-C D$ allows extension of the applications as the method of preparation of the inclusion compounds is easier compared to native $\beta-C D$.

The inclusion complexation behaviour, characterization, and binding ability of herbicide MCPA with $\beta-C D$, Me- $\beta-C D$ and HP- $\beta-$ $C D$ were investigated. The phase solubility study showed that in all cases $\beta$-CDs increased the water solubility of the herbicide and the inclusion complexes have been formed in a stoichiometric ratio of $1: 1$. Stability constants were calculated from the phase solubility diagrams and indicated the following trend: Me- $\beta-\mathrm{CD} \approx \mathrm{HP}-$ $\beta-C D>\beta-C D$. NMR studies were also accomplished for the prepared solid systems and the data gathered confirm the formation of the MCPA/HP- $\beta-C D$ and MCPA/Methyl- $\beta-C D$ inclusion complexes. The complex stoichiometric ratio of both systems was of $1: 1$. Computer models on functionalized $\beta$-CDs showed also an increased binding affinity for MCPA compared to pure $\beta-C D$ accounting for these experimental findings.

This inclusion complexation could be regarded as an important step in the design of novel formulations of MCPA with enhanced chemical properties.

\section{Aknowledgments}

Financial support from Fundacão para a Ciência e Tecnologia FCT/MCTES project PTDC/AGRAAM/105044/2008, National Funds PIDDAC also co-financed by the European Community Fund FEDER through COMPETE - Programa Operacional Factores de Competitividade (POFC), is gratefully acknowledged. F. Cagide (SFRH/BPD/ $74491 / 2010)$ thanks FCT grant. MMF acknowledges support by the Portuguese "Fundação para a Ciência e a Tecnologia" through the program Ciência 2008 and contract PEst-OE/EEI/UI0752/2011 and resources from the project SeARCH (Services and Advanced Research Computing with HTC/HPC clusters) funded under contract CONC-REEQ/443/2005. MMF wished to thank J.W. Ponder for help with tinker and W.L. Jorgensen for providing us with recent OPLS parameters.

\section{Appendix A. Supplementary material}

Supplementary data associated with this article can be found, in the online version, at http://dx.doi.org/10.1016/j.molstruc.2013. 12.067. These data include MOL files and InChiKeys of the most important compounds described in this article.

\section{References}

[1] Food and Agriculture Organization of the United Nations, FAO statistical yearbook 2013: World food and agriculture. Rome, 2013.

[2] S.S.S. Saravi, M. Shokrzadeh, Role of pesticides in human life in the modern age: A review, in: M. Stoytcheva (Ed.), Pesticides in the Modern World - Risks and Benefits, InTech Rijeka, Croatia, 2011, pp. 3-12.

[3] M.G. Mogul, H. Akin, N. Hasirci, D.J. Trantolo, J.D. Gresser, D.L. Wise, Resour. Conserv. Recy. 16 (1-4) (1996) 289-320.

[4] G.B. Beestman, Controlled release in crop protection: Past experience and future opportunities, in: G. Voss, G. Ramos (Eds.), Chemistry of Crop Protection, Wiley, New York, 2003, pp. 272-279.

[5] F. Sopeña, C. Maqueda, E. Morillo, Cien. Inv. Agr. 36 (2009) 27-42.

[6] S. Dubey, V. Jhelum, P.K. Patanjali, J. Sci. Ind. Res. 70 (2011) 105-112.

[7] M.J. Gimeno, J. Environ. Sci. Health B 31 (3) (1996) 407-420.

[8] D.K. Rodham, Curr. Opin. Colloid Interface Sci. 5 (2000) 280-287.

[9] E.M. Garrido, M. Santos, P. Silva, F. Cagide, J. Garrido, F. Borges, J. Environ. Sci. Health B 47 (2012) 869-875.

[10] F. Shahidi, X.-Q. Han, Crit. Rev. Food Sci. Nutr. 33 (6) (1993) 501-547.

[11] H. Dodziuk, Cyclodextrins and Their Complexes: Chemistry, Analytical Methods, Applications, Wiley-VCH, Weinheim, Germany, 2006.

[12] Eurostat, The use of plant protection products in the European Union: Data 1992-2003, Office for official publications of the European Communities, Luxembourg, 2007.

[13] T. Kiely, D. Donaldson, A. Grube, Pesticides industry sales and usage - 2000 and 2001 market estimates. U.S. Environmental Protection Agency, Washington DC, USA, 2004.

[14] V. Triantafyllopoulou, D. Mentzafos, F. Tsorteki, Kostas Bethanis, J. Chem. Crystallogr. 42 (3) (2012) 238-244.

[15] C. Costa, S. Maia, P. Silva, J. Garrido, F. Borges, E.M. Garrido, Int. J. Photoenergy, 2013, pp. 8 (Article ID 542783).

[16] T. Higuchi, K.A. Connors, Adv. Anal. Chem. Instrum. 4 (1965) 117-212.

[17] A. Topalov, B. Abramovic, D. Molnár-Gábor, J. Csanádi, O. Arcson, J. Photochem. Photobiol. A: Chem. 140 (3) (2001) 249-253.

[18] J.W. Ponder, F.M. Richards, J. Comput. Chem. 8 (7) (1987) 1016-1024.

[19] W.L. Jorgensen, D.S. Maxwell, J. Tirado-Rives, J. Am. Chem. Soc. 118 (45) (1996) $11225-11236$

[20] W.L. Jorgensen, J. Chandrasekhar, J.D. Madura, R.W. Impey, M.L. Klein, J. Chem. Phys. 79 (2) (1983) 926-935.

[21] D. Qiu, P.S. Shenkin, F.P. Hollinger, W.C. Still, J. Phys. Chem. A 101 (16) (1997) 3005-3014.

[22] T. Hou, J. Wang, Y. Li, W. Wang, J. Chem. Inf. Model. 51 (1) (2011) 69-82.

[23] T. Loftsson, P. Jarho, M. Másson, T. Järvinen, Exp. Opin. Drug Deliv. 2 (2) (2005) 335-351.

[24] C. Yáñez, P. Cañete-Rosales, J.P. Castillo, N. Catalán, T. Undabeytia, E. Morillo, PLoS ONE 7 (8) (2012) e41072.

[25] H.-J. Schneider, F. Hacket, V. Rüdiger, H. Ikeda, Chem. Rev. 98 (5) (1998) 17551786.

[26] B. Yang, J. Lin, Y. Chen, Y. Liu, Bioorg. Med. Chem. 17 (17) (2009) 6311-6317.

[27] W.S. Cai, T. Wang, Y.Z. Liu, P. Liu, C. Chipot, X.G. Shao, Curr. Org. Chem. 15 (6) (2011) 839-847.

[28] C. Parthier, M. Kleinschmidt, P. Neumann, R. Rudolph, S. Manhart, D. Schlenzig, J. Fanghanel, J.-U. Rahfeld, H.-U. Demuth, M.T. Stubbs, Proc. Natl. Acad. Sci. USA 104 (35) (2007) 13942-13947.

[29] J.J. Stezowski, W. Parker, S. Hilgenkamp, M. Gdaniec, J. Am. Chem. Soc. 123 (17) (2001) 3919-3926.

[30] Y. Yu, C. Chipot, W. Cai, X. Shao, J. Phys. Chem. B 110 (12) (2006) 6372-6378. 